\title{
Article \\ Theoretical Investigation on the Friction Behavior of Bio-Inspired Hard-Soft-Integrated Materials
}

\author{
Mi Wang ${ }^{1,2}$, Wei Yang ${ }^{1}$, Hao Cui ${ }^{2}$, Shu-Chen Yang ${ }^{1}$, Zhen-Ning Liu ${ }^{2}$ and Guo-Long Lu ${ }^{2, *}$ \\ 1 Engineering College, Changchun Normal University, Changchun 130032, China; \\ wangmi003@ccsfu.edu.cn (M.W.); QZ202020020@stu.ccsfu.edu.cn (W.Y.); ysc2017@mail.cncnc.edu.cn (S.-C.Y.) \\ 2 Key Laboratory for Bionic Engineering (Ministry of Education), Jilin University, Changchun 130022, China; \\ cuihao1817@mails.jlu.edu.cn (H.C.); liu_zhenning@jlu.edu.cn (Z.-N.L.) \\ * Correspondence: guolonglu@jlu.edu.cn; Tel.: +86-0431-85095760
}

Citation: Wang, M.; Yang, W.; Cui, H.; Yang, S.-C.; Liu, Z.-N.; Lu, G.-L. Theoretical Investigation on the Friction Behavior of Bio-Inspired Hard-Soft-Integrated Materials. Coatings 2021, 11, 1296. https:// doi.org/10.3390/coatings11111296

Academic Editor: Diego MartinezMartinez

Received: 29 August 2021

Accepted: 22 October 2021

Published: 26 October 202

Publisher's Note: MDPI stays neutral with regard to jurisdictional claims in published maps and institutional affiliations.

Copyright: (c) 2021 by the authors. Licensee MDPI, Basel, Switzerland. This article is an open access article distributed under the terms and conditions of the Creative Commons Attribution (CC BY) license (https:// creativecommons.org/licenses/by/ $4.0 /)$.

\begin{abstract}
Structural biological materials with integrated soft and hard phases are ubiquitous in nature. Over recent decades, bio-inspired hard-soft-integrated materials (BHSIMs) have shown excellent mechanical properties of drag reduction and abrasion resistance. This work is proposed to investigate the friction behaviors of BHSIMs via theoretical modeling, numerical simulation and experimental verification. First, the mathematical model of the friction process was established based on the classic adhesive friction theory. Then, a range of factors in the friction process were examined by simulation and the respective friction coefficients were discussed. Subsequently bio-inspired materials with integrated soft and hard layers were prepared by 3D printing and their friction coefficients were measured by experiments, which had verified the results of theoretical analyses.
\end{abstract}

Keywords: hard-soft integrated; friction behavior; bio-inspired material; friction coefficient; 3D printing

\section{Introduction}

It is well known that structural biological materials are often of heterogeneous phases and hierarchical architectures, which afford outstanding mechanical performance to protect an organism against complex environments [1-3]. One particular feature of these structural biomaterials, such as nacre, bones and skins, is the integration of periodic soft and hard layers, which widely exist in a vast array of invertebrates and vertebrates [4]. For example, shark skin consists of stiff surface denticles embedded in a supporting layer of flexible collagenous matrix [5]. Snake skin also exhibits a similar arrangement of relative stiff scale supported by a flexible layer $[5,6]$. It has been shown that biomaterials with integrated hard and soft phases are of exceptional mechanical properties beyond those of pure soft or hard phase, demonstrating a remarkable balance of stiffness, strength, fracture toughness, energy dissipation and wear resistance [7-14]. It is commonly assumed that in such a scenario, the hard phase can improve the hardness of materials and resist deformation and wear, whereas the soft phase can function to dissipate and absorb external energy. Although nature has evolved abundant solutions to achieve low friction and wear reduction, it remains a challenge to design and fabricate materials with desired friction and wear behavior based on the integrated structure of soft and hard components [15]. Herein, we have investigated the friction behavior of bio-inspired hard-soft-integrated materials (BHSIMs) under dry sliding conditions via a theoretical approach of mathematical modeling and simulation. Then, a series of BHSIMs with varied contents of hard and soft phases have been prepared by $3 \mathrm{D}$ printing, and the friction properties of the resultant specimens are examined by experiment. 


\section{Theory}

In order to investigate the friction behavior of BHSIMs, we first set up a mathematical model based on adhesive friction. It is widely believed that adhesion between surfaces is the main source of friction and surface roughness plays a secondary role based on the classic adhesive friction theory [16] (surface roughness decreases the "real area of contact", thereby reducing the adhesion and consequently the friction between surfaces).

The friction behavior of BHSIMs can be explained by the adhesion theory of friction [17]. In the sliding process, normal load can be expressed as

$$
W=A_{s} q_{s}+A_{h} q_{h}
$$

where $A_{s}$ and $A_{h}$ are the "real areas of contact" for soft and hard phases, respectively, and $q_{s}$ and $q_{h}$ are force on unit area of the interacting surfaces for soft and hard phases.

The adhesive friction of BHSIMs is a complex trait combining the individual properties of soft and hard phases, but also with mutual influence between these two phases ("real area of contact" of soft and hard phases in sliding process will influence each other). For BHSIMs here, we describe it by two scalar parameters, Young's modulus $E_{s}$ (Young's modulus of soft phase) and $E_{h}$ (Young's modulus of hard phase), which are the load per unit surface per relative elongation/compression of the chain for pure soft and hard phases. When a normal load is applied to the BHSIMs, the deformations of hard phase and soft phase per relative compression have to be similar within the sliding process, as shown in Figure 1, which results in a change in "real area of contact", thus leading to the redistribution of the normal load.

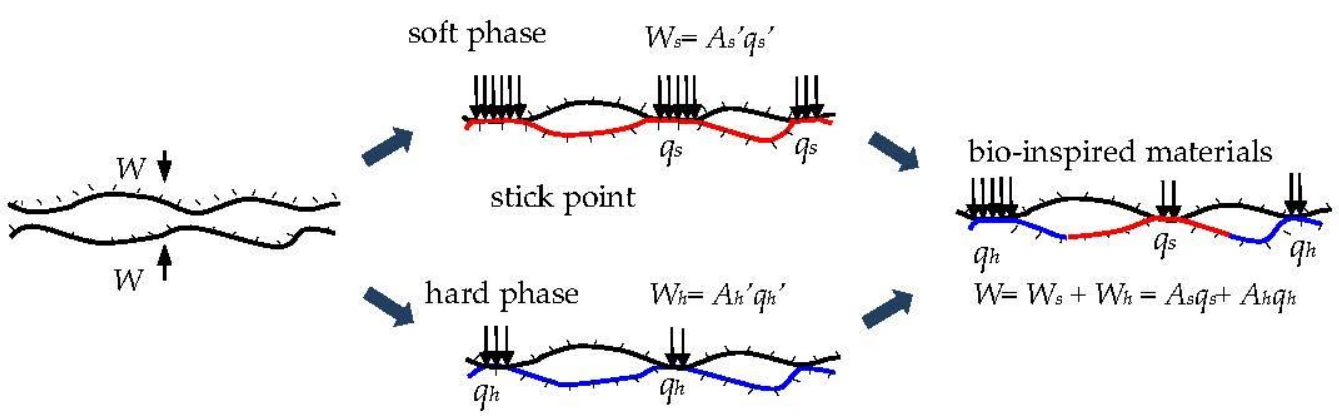

Figure 1. Schematic illustration for the rubbing interface of bio-inspired hard-soft-integrated materials (BHSIMs).

To deduce the change in the friction coefficient from $E_{s}$ and $E_{h}$, we have designated the volume ratios of soft and hard phases in BHSIMs as $c \%$ and $1-c \%$. Then, based on the theory of statistics and the structure characteristic of the combined soft and hard phases, the normal force can be given according to a simple equation:

$$
W=W_{s}+W_{h}=c \% A E_{s} \varepsilon+(1-c \%) A E_{h} \varepsilon
$$

where $A$ denotes the "real area of contact" of BHSIMs, $\varepsilon$ is the strain on the adhesion point.

Hence, the sliding process can be considered as the formation and destruction of the adhesion point. Friction force is therefore expressed as:

$$
F=c \% A \tau_{s}+(1-c \%) A \tau_{h}
$$

where $\tau_{s}$ and $\tau_{h}$ are the shear strength for soft phase and hard phase, respectively.

With respect to Coulomb friction, the dynamic friction coefficient $f$ is described by the friction force $F$ divided by the normal force $W$, then, the friction coefficient $f$ can be determined by:

$$
f=\frac{c \% f_{s} E_{s}+(1-c \%) f_{h} E_{h}}{c \% E_{s}+(1-c \%) E_{h}}
$$


Equation (4) indicates that the friction coefficient of BHSIMs is a parameter that relates to Young's modulus and content of soft and hard phase.

\section{Simulation}

In order to gain more insight into the structure-property relationship in BHSIMs, finite element (FE) models of the sliding process of BHSIMs were established. It is believed that the adhesion between surfaces is the main source of friction and surface roughness plays a secondary role. In order to simplify the model, we are not concerned with such roughness in simulation. Thus, we assume that the surfaces of the friction pair are of geometrically simple and smooth shapes. Figure 2a is the FE model of the sliding process of BHSIMs, which is based on ABAQUS/Explicit. In this model, surface-to-surface contact mode was used to simulate the friction boundary condition between the bio-inspired materials and rubbing pin. The friction tool is set as a rigid body, the BHSIMs is composed of layered materials with different soft and hard phases (different elastic modulus). The size of the soft and hard phase layered bionic material during simulation is $5 \mathrm{~mm} \times 5 \mathrm{~mm} \times 6 \mathrm{~mm}$ and the thickness of the soft/hard phase layer (C-S/C-H) is $0.2 \mathrm{~mm}$. The BHSIMs is meshed with a C3D8R element which is evaluated using hourglass control elements and reduced integration. The friction tool is located above the material surface at $0 \mathrm{~s}$. It then brings contact with the material surface at $0.1 \mathrm{~s}$, and then moved alternately. The Mises stress distributions of bio-inspired materials at different timepoints in the sliding process are shown in Figure 2b. It can be observed that the contact zone has the maximum Mises stress, and the Mises stress decreases dramatically beyond the deformation zone. More importantly, the maximum Mises stress occurs at the hard phase layer, whereas relatively smaller Mises stresses can be found at the soft phase layer. The observation of more concentrated stress on the hard phase is probably due to the remarkable load carrying capability of the hard phase, and indicates a redistribution of the normal load from the soft phase to hard phase in sliding process.

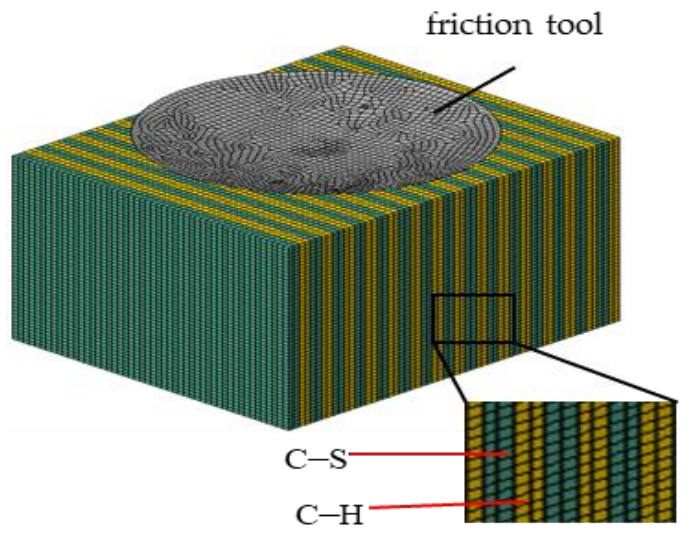

(a)
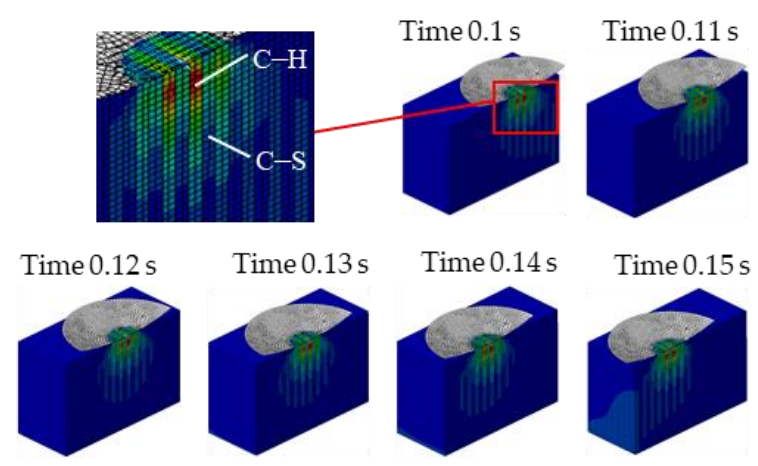

Time $0.13 \mathrm{~s}$

Time $0.14 \mathrm{~s}$

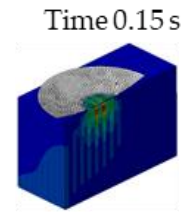

(b)

Figure 2. Finite element (FE) model of dry sliding process of BHSIMs. (a) FE model of BHSIMs, (b) Mises stress distributions sliding process at different timepoints.

To better understand the effect of Young's modulus of hard phases on the friction coefficients, a series of BHSIMs simulations were carried out and separate sets of data were obtained, in which the $E_{h}$ was the unique variable ranging from 400 to $3000 \mathrm{MPa}$. The insets in Figure $3 \mathrm{~b}$ is the variables and constants in the simulation ( $E_{s}$ was selected for $270 \mathrm{MPa}$, and the friction coefficients of the soft and hard phase are 0.1 and 0.2 , respectively). It can be observed in Figure 3a that the friction force on the hard phase (shaded area), as well as the total friction force (black line), increases as the $E_{h}$ rises. Meanwhile, the friction force on the soft phase decreases with the increase of $E_{h}$. It is also found that the increase of $E_{h}$, also causes a redistribution in line with the findings from Figure 2, i.e., the higher the 
$E_{h}$, the more load on the hard phase (inset in Figure 3a). Figure $3 \mathrm{~b}$ shows the relationship between the load on the hard phase and the friction coefficient based on the simulated output result, it can be observed that, with the $E_{h}$ varying from $700 \mathrm{MPa}$ to $3000 \mathrm{MPa}$, the friction coefficient of the BHSIMs linearly increases from 0.142 to 0.189 , which can be interpreted by Equation (4).

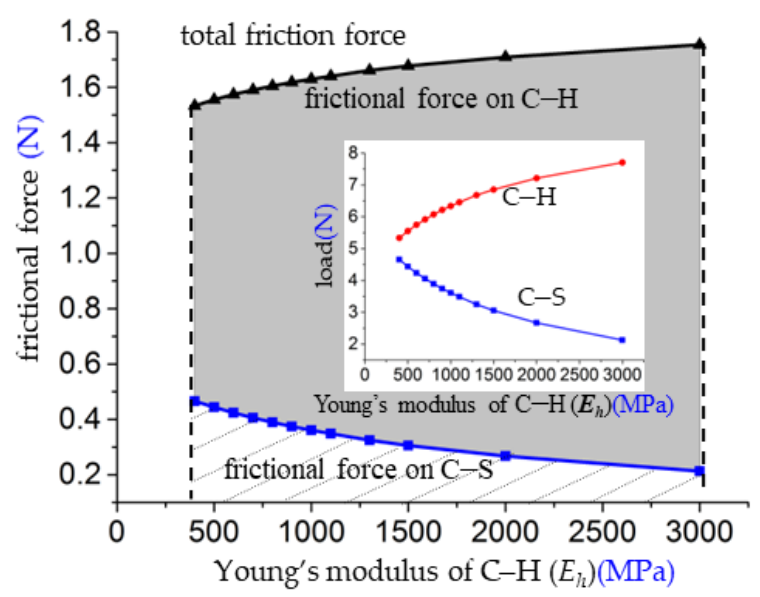

(a)

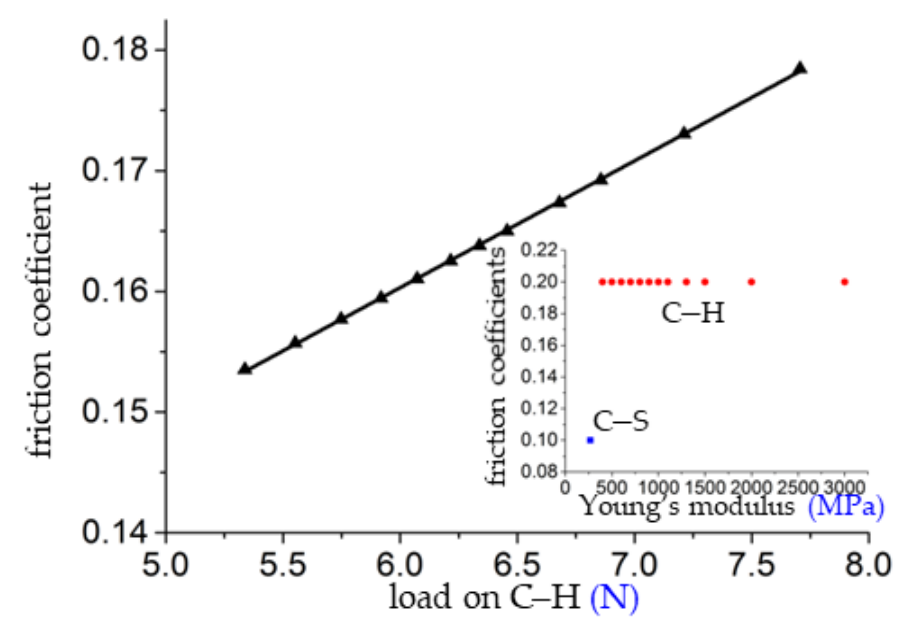

(b)

Figure 3. Curves of friction force and friction coefficient for BHSIMs with varied elastic modulus of hard phase $\left(E_{h}\right)$. (a) friction force (inset is the load); (b) friction coefficient (inset is the friction coefficient).

Next, in order to investigate the effect of the friction coefficient of hard material components on the friction properties of BHSIMs, a range of bio-inspired hard-soft-integrated materials with different material components friction coefficients were established and related data were obtained, in which the hard phase friction coefficient was the unique variable varied from 0.17 to 0.25 . The insets in Figure $4 \mathrm{~b}$ are the variables and constants in the simulation ( $E_{s}$ was selected for $270 \mathrm{MPa}, E_{h}$ was selected for $700 \mathrm{MPa}$, and the friction coefficient of the soft phase was selected for 0.1). The results are shown in Figure 4. It is found that increasing the friction coefficient of the hard phase incurs an increasing friction force in the sliding process (Figure $4 \mathrm{a}$ ), which leads to a linearly increasing friction coefficient of BHSIMs from 0.17 to 0.25 (Figure $4 \mathrm{~b}$ ). These results are also in line with the calculated values from Equation (4).

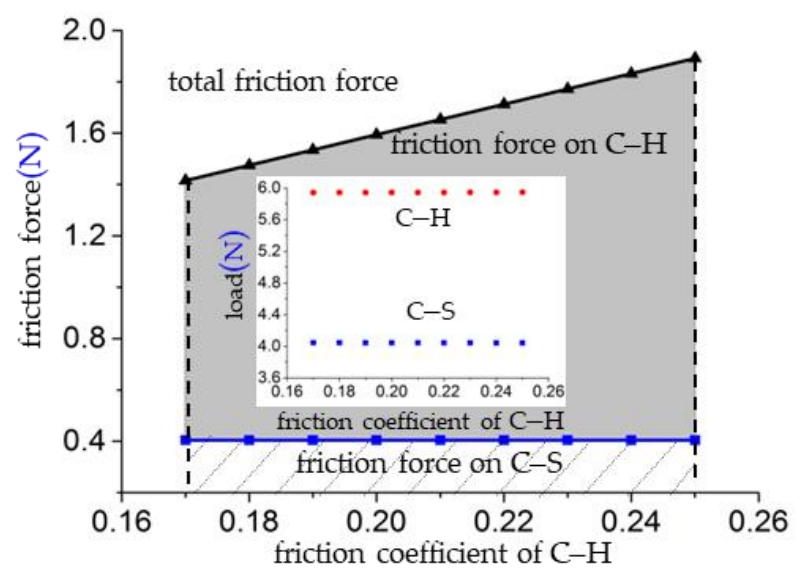

(a)

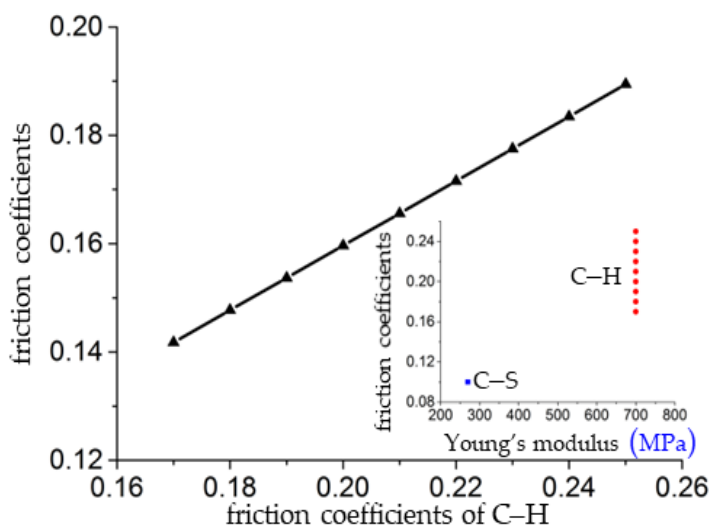

(b)

Figure 4. Curves of friction force and friction coefficient for BHSIMs with varied friction coefficients of hard phase. (a) friction force (inset is the load); (b) friction coefficient (inset is the friction coefficient). 


\section{Experiment}

Subsequently, in order to test our simulated results, a series of composites with integrated hard and soft phases have been fabricated by 3D printing from two starting materials (VisiJet CR-WT and VisiJet CF-BK are the basic materials equipped with 3D printers (ProJet 5500X, 3D SYSTEMS, Hangzhou, China)) with an elastic modulus differential (Table 1).

Table 1. Properties of the materials to make the composites with integrated hard and soft phases.

\begin{tabular}{cccc}
\hline Content & Density $\left(\mathbf{g} / \mathrm{cm}^{3}\right)$ & Young's Modulus (MPa) & Tensile Strength (MPa) \\
\hline VisiJet CR-WT & 1.18 & $1000-1600$ & $37-47$ \\
VisiJet CF-BK & 1.12 & $0.27-0.43$ & $0.2-0.4$ \\
\hline
\end{tabular}

3D printing allows an easy fabrication of objects with complex geometries, making it a viable option for direct manufacturing. Thus, we have envisioned that $3 \mathrm{D}$ printing can be an attractive fabrication technique for the preparation of BHSIMs. Photographs of printed specimens are shown in Figure 5a, which verifies that BHSIMs can be readily achieved by a well-controlled 3D printing process. Herein, five compositions of printing material (namely, C100, C150, C200, C250 and C300 with Young's modulus decreasing from C100 to C300) have been prepared by mixing different ratios of VisiJet CR-WT and VisiJet CF-BK, which represent the hard and soft components of the bio-inspired materials, respectively. Subsequently, a range of bio-inspired materials with integrated hard and soft layers $(0.2 \mathrm{~mm})$ have been fabricated by 3D printing. Specifically, we prepared a series of soft and hard layered bionic materials with a width of $15 \mathrm{~mm}$, length of $30 \mathrm{~mm}$, and thickness of $2 \mathrm{~mm}$. As shown in Figure 5b, we define the stripe width of the soft material as "a", and the stripe width of the hard material as " $\mathrm{b}$ ". As the color gradually gets darker from $\mathrm{C} 100$ to $\mathrm{C} 300$, the color of composts also changes from the upper left (C100/C100) to lower right $(\mathrm{C} 300 / \mathrm{C} 300)$.

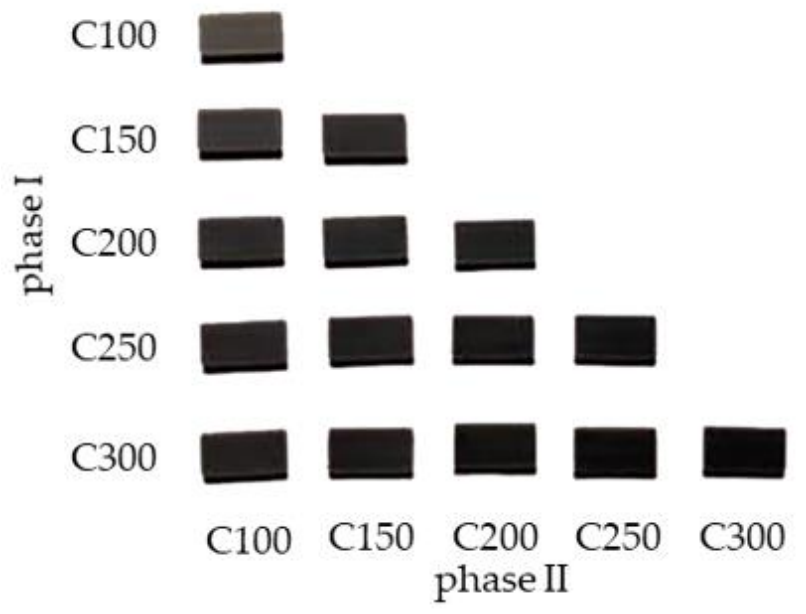

(a)

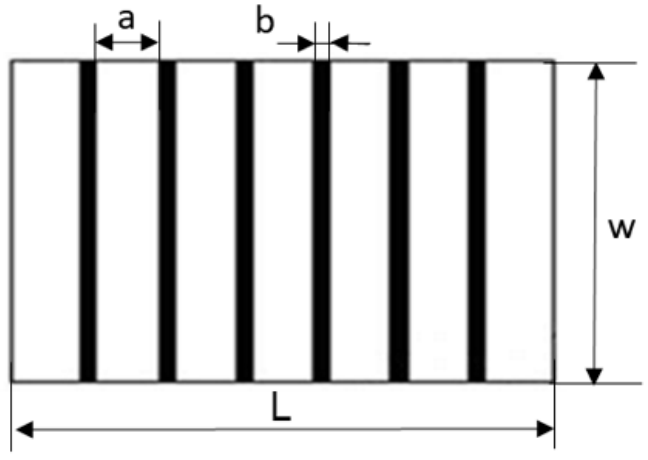

(b)

Figure 5. (a) Photographs of the 3D printed specimens for the tribological experiment; (b) the schematic diagram of soft and hard layered bionic materials.

To corroborate the aforementioned findings, the friction coefficients of BHSIMs with different configurations of soft and hard phases were measured on a micro-tribotester. The friction coefficient can be measured directly via the MG-2000 high temperature and high speed friction and wear tester (Kehua Test Machine Manufacturing Company, Hebei, China). In the test, we choose a metal ball with a $10 \mathrm{~mm}$ diameter as the counter-grinding part. Moreover, the normal phase load of the metal ball to the layered bionic material is set to $10 \mathrm{~N}$, the sliding distance of the metal ball is $2 \mathrm{~mm}$, the frequency is $10 \mathrm{~Hz}$, and the test 
time is $10 \mathrm{~min}$. We set the metal ball reciprocating movement, which is from top to bottom and then from bottom to top. In order to verify the validity of the relationship between the friction coefficient of BHSIMs and the friction coefficients of hard and soft phases, based on the classic adhesive friction theory, surface roughness in the experiment is not focused on. Thus, we assume that the surfaces of the friction pair are of geometrically simple and smooth shapes. To our delight, the friction coefficients of bio-inspired materials indeed reveal a trend depending on the composition of the material. The friction coefficient of BHSIMs increases as the elastic modulus of hard materials increase because the friction coefficient of hard materials is set to be greater than that of soft materials during the experiment. Then during the friction process, materials with larger elastic modulus bear more load. The greater the elastic modulus of hard materials, the closer the friction factor of BHSIMs is to the friction factor of the hard materials (0.2), which conforms to the law shown in Equation (4).

Specifically, as the friction coefficient of the constituting materials increases from C100 to C300, the friction coefficient of the bio-inspired composite material increases from C100/C100 to C300/C300 (Figure 6). It can be seen from Figure 6 that the friction coefficient of the soft and hard layered bionic material will change as the material properties of the hard material components change, which indicates that the elastic modulus $E_{s}$ and $E_{h}$, the friction coefficients $f_{s}$ and $f_{h}$ of the hard layered bionic materials in the soft and hard materials are the main factors affecting the friction coefficient of the soft and hard layered bionic materials. The experimental results coincide with Equation (4).

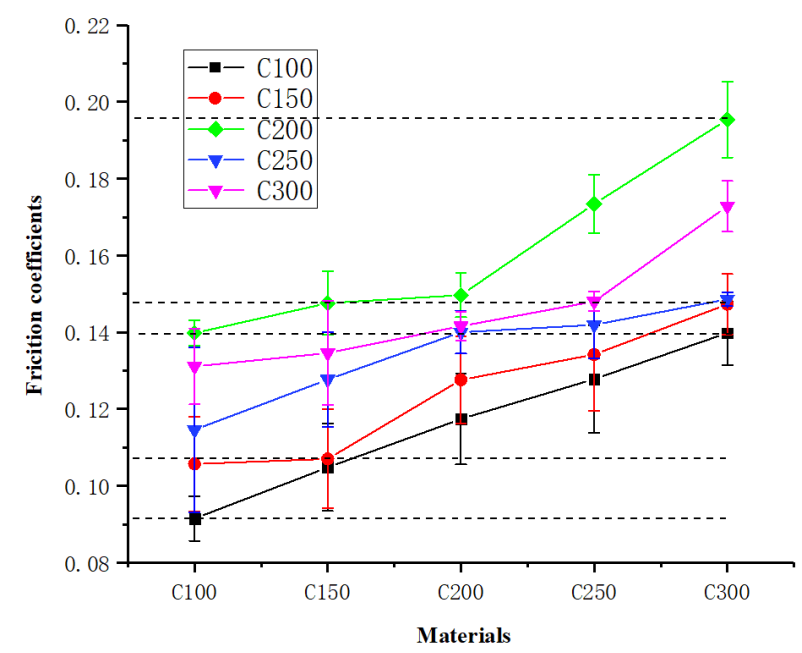

Figure 6. Friction coefficients of BHSIMs (phase I is labeled on the X-axis and phase II is indicated by legends).

\section{Conclusions}

In this work, we have investigated the friction behaviors of bio-inspired hard-softintegrated materials (BHSIMs) via theoretical modeling, numerical simulation and experimental verification. Theoretical analysis reveals that the friction coefficient of BHSIMs is a parameter correlating to the Young's modulus and friction coefficients of both the soft phase and hard phase. Through the numerical simulation, the Mises stress distribution, as well as its time-course variation, is discussed to prove the methods. The simulation results of a series of BHSIMs show that the friction coefficient of bio-inspired material lies in between the friction coefficients of the hard phase and soft phase, and the increase of the friction coefficient of the hard phase leads to the increase of the overall friction coefficient of BHSIMs (linearly increasing friction coefficients of BHSIMs from 0.17 to 0.25 ). The larger the elastic modulus of the hard phase is, the closer the friction coefficient of BHSIMs to the friction coefficient of the hard phase is (with the $E_{h}$ varying from $700 \mathrm{MPa}$ to $3000 \mathrm{MPa}$, the friction coefficient of the BHSIMs linearly increases from 0.142 to 0.189 ). Experimental results verify the validity of the relationship between the friction coefficient 
of BHSIMs and the friction coefficients of the hard and soft phases (when the friction coefficient of the constituting materials increases from C100 to C300, the friction coefficient of the bio-inspired composite material increases from C100/C100 to C300/C300).

Author Contributions: Conceptualization, M.W. and W.Y.; methodology, G.-L.L.; software, M.W.; validation, H.C. and Z.-N.L.; formal analysis, M.W.; investigation, W.Y.; resources, S.-C.Y. and G.-L.L.; data curation, W.Y.; writing—original draft preparation, M.W.; writing—review and editing, W.Y.; visualization, H.C. and Z.-N.L.; supervision, S.-C.Y. and G.-L.L.; project administration, H.C.; funding acquisition, Z.-N.L. All authors have read and agreed to the published version of the manuscript.

Funding: This research was funded by the National Natural Science Foundation of China (51975245 and 52075214). Funding support also came from the Jilin Provincial Science \& Technology Department (20200201058JC, 20190303039SF and 20200201224JC), Key Science and Technology R\&D Projects of Jilin Province (2020C023-3), Program of Jilin University Science and Technology Innovative Research Team (2020TD-03), and Youth Development Program of Jilin University (2020-JCXK-22).

Institutional Review Board Statement: Not applicable.

Informed Consent Statement: Not applicable.

Data Availability Statement: Not applicable.

Conflicts of Interest: The authors declare no conflict of interest. The funders had no role in the design of the study; in the collection, analyses, or interpretation of data; in the writing of the manuscript, or in the decision to publish the results.

\section{References}

1. Yang, L.; Larouche, N.; Chenitz, R.; Zhang, G.; Lefèvre, M.; Dodelet, J.-P. Activity, performance, and durability for the reduction of oxygen in pem fuel cells, of Fe/N/C electrocatalysts obtained from the pyrolysis of metal-organic-framework and iron porphyrin precursors. Electrochim. Acta 2015, 159, 184-197. [CrossRef]

2. Chen, H.-Y.; Wang, P.-Y. Special Issue: Biointerface coatings for biomaterials and biomedical applications. Coatings 2021, 11, 423. [CrossRef]

3. Li, H.; Chen, W.; Shi, H.; Zhang, C.; Liu, X.; Zhong, L. Basic bio-tribological performance of insulating $\mathrm{Si}_{3} \mathrm{~N}_{4}$-based ceramic as human body replacement joints. Coatings 2021, 11, 938. [CrossRef]

4. Meyers, M.A.; McKittrick, J.; Chen, P.Y. Structural biological materials: Critical mechanics-materials connections. Science 2013, 339, 773-779. [CrossRef] [PubMed]

5. Filippov, A.; Gorb, S.N. Frictional-anisotropy-based systems in biology: Structural diversity and numerical model. Sci. Rep. 2013, 3, 1240. [CrossRef]

6. Klein, M.C.; Deuschle, J.K.; Gorb, S.N. Material properties of the skin of the Kenyan sand boa Gongylophis colubrinus (Squamata, Boidae). J. Comp. Physiol. A 2010, 196, 659-668. [CrossRef] [PubMed]

7. Wegst, U.G.; Bai, H.; Saiz, E.; Tomsia, A.P.; Ritchie, R.O. Bioinspired structural materials. Nat. Mater. 2015, 14, 23-36. [PubMed]

8. Chen, P.Y.; Lin, A.Y.; Lin, Y.S.; Seki, Y.; Stokes, A.G.; Peyras, J.; Olevsky, E.A.; Meyers, M.A.; McKittrick, J. Structure and mechanical properties of selected biological materials. J. Mech. Behav. Biomed. Mater. 2008, 1, 208-226. [CrossRef] [PubMed]

9. Meyers, M.A.; Chen, P.-Y.; Lin, A.Y.-M.; Seki, Y. Biological materials: Structure and mechanical properties. Prog. Mater. Sci. 2008, 53, 1-206. [CrossRef]

10. Wang, L.; Boyce, M.C. Bioinspired structural material exhibiting post-yield lateral expansion and volumetric energy dissipation during tension. Adv. Funct. Mater. 2010, 20, 3025-3030. [CrossRef]

11. Ortiz, C.; Boyce, M.C. Materials science. Bioinspired structural materials. Science 2008, 319, 1053-1054. [CrossRef] [PubMed]

12. Li, H.; Yue, Y.; Han, X.; Li, X. Plastic deformation enabled energy dissipation in a bionanowire structured armor. Nano Lett. 2014, 14, 2578-2583. [CrossRef] [PubMed]

13. Yao, H.; Xie, Z.; He, C.; Dao, M. Fracture mode control: A bio-inspired strategy to combat catastrophic damage. Sci. Rep. 2015, 5, 8011. [CrossRef] [PubMed]

14. Huang, Z.; Pan, Z.; Li, H.; Wei, Q.; Li, X. Hidden energy dissipation mechanism in nacre. J. Mater. Res. 2014, $29,1573-1578$. [CrossRef]

15. Fratzl, P.; Kolednik, O.; Fischer, F.D.; Dean, M.N. The mechanics of tessellations-Bioinspired strategies for fracture resistance. Chem. Soc. Rev. 2016, 45, 252-267. [CrossRef] [PubMed]

16. Bharat, B. Introduction to Tribology, 2nd ed.; John Wiley \& Sons: Hoboken, NJ, USA, 2013.

17. Bowden, F.P.; Tabor, D.; Palmer, F. The friction and lubrication of solids. Am. J. Phys. 1951, 19, 428-429. [CrossRef] 